Hot Topics

Z Rheumatol 2020 $\cdot 79: 554-561$

https://doi.org/10.1007/s00393-020-00822-2

Online publiziert: 29. Mai 2020

(C) Der/die Autor(en) 2020

\section{Redaktion}

U. Müller-Ladner, Bad Nauheim

U. Lange, Bad Nauheim

\author{
M. Krusche ${ }^{1} \cdot$ N. Ruffer ${ }^{2}$ M. Grahammer ${ }^{3}$ J. Knitza ${ }^{4}$ \\ ${ }^{1}$ Medizinische Klinik mit Schwerpunkt Rheumatologie und Klinische Immunologie, Charité \\ Universitätsmedizin, Berlin, Deutschland \\ ${ }^{2}$ Universitätsklinikum Hamburg-Eppendorf, Hamburg, Deutschland \\ ${ }^{3}$ ABATON GmbH, Berlin, Berlin, Deutschland \\ ${ }^{4}$ Medizinische Klinik 3 - Rheumatologie und Immunologie, Universitätsklinikum Erlangen, Friedrich- \\ Alexander-Universität Erlangen-Nürnberg (FAU), Erlangen, Deutschland
}

\title{
Apps und ihre Anwendungsgebiete in der Rheumatologie
}

zu tragen [5]. Auch die neu gegründete Kommission „Digitale Rheumatologie“ der deutschen Gesellschaft für Rheumatologie (DGRh) setzt sich verstärkt mit der Thematik auseinander.

\section{Potenzial von Apps}

Je nach Zielgruppe (Patient und/oder Arzt) und Zeitpunkt der medizinischen Behandlung bestehen verschiedene Anwendungsmöglichkeiten für Apps in der Rheumatologie. Der Patient könnte niedrigschwelliger und schneller erste Diagnosevorschläge, relevante Informationen und schließlich einen Abklärungstermin erhalten [6, 7]. Nach Diagnosestellung ermöglichen Apps, die Verträglichkeit und Wirksamkeit der Therapien objektiv und kontinuierlich zu monitorieren $[8,9]$. Diese wertvollen Daten könnten Patienten auf freiwilliger Basis der Forschung zur Verfügung stellen, um kontinuierlich die Versorgung $\mathrm{zu}$ verbessern. Komorbiditäten könnten ebenfalls niedrigschwelliger erkannt und behandelt werden. Außerdem könnten Apps dazu beitragen, zeitintensive Patientenschulungen und Rehabilitationen effizienter zu gestalten. Fachwissen kann ebenfalls per App digital Patienten und medizinischem Fachpersonal zielgruppengerecht aufbereitet bereitgestellt werden. In den folgenden Abschnitten erhalten Sie konkrete Beispiele für Anwendungsmöglichkeiten in der Rheumatologie.

\section{Definitionen}

Da die Begrifflichkeiten "GesundheitsApp“, „Medizin-App“, „App als Medizinprodukt“ bzw. „digitale Gesundheitsanwendung" häufig unscharf oder auch synonym verwendet werden, sollen die Begriffe definiert werden [10]:

Gesundheits-Apps (englisch: „health app") richten sich im Allgemeinen an gesunde Nutzer, die mithilfe einer App die eigene Gesundheitsförderung (im Sinne einer Primärprophylaxe) betreiben wollen. Sie dienen v. a. der Selbstvermessung und Selbstoptimierung ihrer Nutzer.

Medizin-Apps (englisch: „medical app“) richten sich an Patienten oder Angehörige. Im Fokus dieser Apps steht es, die „Selbstbefähigung" von Betroffenen im Krankheitsmanagement zu stärken (Sekundär- oder Tertiärprophylaxe). Dosisrechner und Nachschlagewerke, die von medizinischem Fachpersonal verwendet werden, werden ebenfalls in dieser Kategorie eingeordnet.

\section{Apps als Medizinprodukte (nach} $\S 3 \mathrm{MPG}$ )

Sowohl Gesundheits-Apps als auch Medizin-Apps können als Medizinprodukte zertifiziert werden, wenn der Hersteller sie mit einer „primären medizinischen Zweckbestimmung" anbietet. Dies ist notwendig, wenn die App die Vermeidung, Diagnose oder Therapie von Krankheiten unterstützen will. 


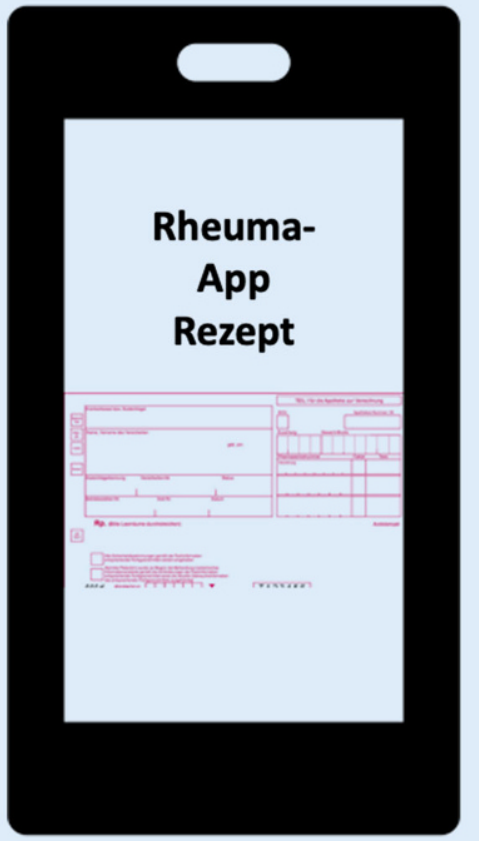

Abb. 1 ॥ Rheuma-App auf Rezept

\section{Digitale Gesundheitsanwendun- gen (nach § 33a SGB V)}

Gemäß der Legaldefinition im Sozialgesetzbuch V (SGB V) sind digitale Gesundheitsanwendungen (DiGAs) Medizinprodukte niedriger Risikoklasse (Klasse I und IIa), die hauptsächlich auf digitalen Technologien beruhen. Sie sollen die Erkennung, Überwachung, Behandlung oder Linderung von Krankheiten, Verletzungen oder Behinderungen unterstützen.

Der Anspruch der Patienten umfasst lediglich Anwendungen, die durch das Bundesamt für Arzneimittel und Medizinprodukte (BfArM) zugelassen und in die Liste gemäß $₫ 139$ e SGB V aufgenommen wurden. Zudem müssen DiGAs vom behandelnden Arzt oder Psychotherapeuten verordnet oder von der Krankenkasse genehmigt werden $(\$ 33 \mathrm{a}$ SGB V). Zu betonen ist auch, dass das Gesetz vorsieht, dass Ärzte für Leistungen, die im Zusammenhang mit einer DiGA stehen, vergütet werden können $(\$ 87$ (5c) SGB V). Aufgrund der hohen Anforderungen an DiGAs und deren Hersteller ist anfangs mit keiner hohen Zahl an DiGAs zu rechnen. Experten gehen von einer Anzahl im knapp zweistelligen Bereich aus.

Der DiGA-Hersteller bestimmt den Indikationsbereich, in welchem DiGAs verordnet bzw. genehmigt werden dürfen. Bevor eine DiGA zugelassen wird, muss diese einen aufwendigen Zulassungsprozess durchlaufen. In diesem Prozess soll zum einen sichergestellt werden, dass die DiGA den Anforderungen an Sicherheit und Funktionstauglichkeit genügt und zum anderen positive Versorgungseffekte bewirkt. Mit positiven Versorgungseffekten meint der Gesetzgeber (1) einen medizinischen Nutzen wie patientenrelevante, therapeutische Endpunkte, aber auch (2) patientenrelevante Verfahrens- und Strukturverbesserungen (pVS). Diese pVS umfassen dabei insbesondere die folgenden Bereiche ( $\$ 8$

(3) DiGA Verordnung [DiGAV]):

- Koordination der Behandlungsabläufe,

- Ausrichtung der Behandlung an Leitlinien und anerkannten Standards,

- Adhärenz,

- Erleichterung des Zugangs zur Versorgung,

- Patientensicherheit,

- Gesundheitskompetenz,

- Patientensouveränität,

- Bewältigung krankheitsbedingter Schwierigkeiten im Alltag oder

- Reduzierung der therapiebedingten Aufwände und Belastungen der Patienten und ihrer Angehörigen.

Vor dem Antragsverfahren beim BfArM muss die DiGA als Medizinprodukt der Klasse I oder IIa zertifiziert werden.

Der Gesetzgeber stellt darüber hinaus noch weitere Anforderungen, die in einem umfassenden Katalog geprüft werden (\$ 3-5 DiGAV; Anlage $1 \& 2$ ebd.): - Sicherheit und Funktionstauglichkeit, - Datenschutz und Datensicherheit,

- Interoperabilität,

- Robustheit,

- Verbraucherschutz,

- Nutzerfreundlichkeit,

- Unterstützung der Leistungserbringer,

- Qualität der medizinischen Inhalte,

- Patientensicherheit.

Zur weiteren Vertiefung kann der „DiGA Leitfaden für Hersteller, Leistungserbrin- ger und Anwender" des BfArM herangezogen werden [11].

\section{Nutzung von Apps unter Patienten und Ärzten}

Die Nutzungsbereitschaft von medizinischen Apps ist unter deutschen Rheumatologe in den letzten Jahren deutlich gestiegen [12]. So berichteten 2018 bereits $49 \%$ der Rheumatologen, medizinische Apps zu verwenden (2016 waren es noch $37 \%$ ). Weiterhin planten im Jahr $201867 \%$ der Rheumatologen die Nutzung von medizinischen Apps in der Routineversorgung (2016: 47\%).

Auch die Verwendung von Social Media im professionellen Kontext ist laut einer Studie des Emerging European League Against Rheumatism Network (EMEUNET) von 2017 unter jungen Rheumatologen weit verbreitet: $71 \%$ der Befragten gaben an, Social Media professionell einzusetzen. Im Vordergrund stehen dabei die Entwicklung eines beruflichen Netzwerkes (79\%) und die persönliche Fortbildung zu bestimmten Forschungsthemen (48\%) [13].

Viele rheumatologische Patienten haben ebenfalls ein großes Interesse, Apps im Kontext ihrer Erkrankung zu verwenden. So ergab eine Patientenumfrage im Jahr 2019, dass 90 \% der befragten Rheumapatienten regelmäßig ein Smartphone nutzten und $65 \%$ den Einsatz von medizinischen Apps als hilfreich ansehen [14]. Interessanterweise wurden medizinische Apps jedoch nur von 6\% der Befragten genutzt. Die Arbeit zeigte außerdem, dass Patienten sich von einer App insbesondere Informationen zur Erkrankung, zu Medikamenten und zur Ernährung wünschen.

Zusätzlich ergab sich bei den Patienten eine große Bereitschaft (96\%), persönliche Daten aus Apps für Forschungszwecke bereitzustellen. In einer britischen Studie an Patienten mit rheumatoider Arthritis (RA) äußerten die Teilnehmer ebenfalls eine hohe Bereitschaft, persönliche App-Daten für Forschungszwecke zur Verfügung zu stellen [15]. 


\section{Konkrete Anwendungs- möglichkeiten von Apps}

Je nach Zielgruppe (Patient und/oder Arzt) der medizinischen App bestehen verschiedene Anwendungsmöglichkeiten für Apps in der Rheumatologie.

\section{Apps zur Diagnoseunterstützung}

Ein anhaltend großes Problem in der Rheumatologie ist der begrenzte Zugang zum Facharzt [16]. Dies ist insbesondere für die Diagnosestellung und eine adäquate Therapieeinleitung von Bedeutung. Andererseits belegen Zahlen, dass bei bis zu zwei Drittel der Erstvorstellungen keine Diagnose einer entzündlich rheumatischen Erkrankung gestellt wird [17]. Verschiedenste App-Lösungen werden daher aktuell erprobt, um den Überweisungsprozess effizienter zu gestalten. So hat z. B. die spanische Fachgesellschaft für Rheumatologie in Kooperation mit der spanischen Fachgesellschaft für Hausärzte eine App entwickelt, die einen klaren Diagnostik- und Überweisungsalgorithmus für Patienten mit muskuloskeletalen Beschwerden vorgibt [18].

Neben dem deutschen Startup Ada Health, deren Patienten-App fachübergreifend Symptome verarbeitet und Verdachtsdiagnosen sowie Handlungsempfehlungen gibt, existieren auch mehrere rheumaspezifische bzw. krankheitsspezifische deutsche Ansätze, wie z.B. RhePort (www.rheport.de) oder Rheu$m a-V O R$ (www.rheuma-vor.de) und internationale Projekte. Ein weiteres Beispiel ist das EU-geförderte Joint Pain Assessment Scoring Tool (JPAST) [19]. Dass die Entwicklung entsprechender Apps jedoch noch in den Kinderschuhen steckt und sicherlich noch Optimierungspotenzial besteht, zeigte sich u. a. in einer englischen Pilotstudie über Symptom-Checker [20]. (Diese erfragen die Symptome und geben potenzielle Diagnosen oder Handlungsanweisungen vor.) Dort wurde bei nur 19\% der Patienten mit rheumatologischen Beschwerden die korrekte Diagnose einer Arthritis gestellt.

Z Rheumatol 2020 · 79:554-561 https://doi.org/10.1007/s00393-020-00822-2

(c) Der/die Autor(en) 2020

\section{Krusche · N. Ruffer · M. Grahammer · J. Knitza}

\section{Apps und ihre Anwendungsgebiete in der Rheumatologie}

\section{Zusammenfassung}

Mit der steigenden Verwendung von Smartphones einhergehend, nimmt auch die Nutzung von mobilen Applikationen (Apps) rapide $z u$. Im medizinischen Kontext könnten chronisch kranke Patienten von dem Einsatz dauerhaft profitieren. Verstärkt wird diese Entwicklung durch das Digitale-VersorgungGesetz (DVG), wonach Patienten ab Q4/2020 einen Rechtsanspruch auf bestimmte Apps, sog. digitale Gesundheitsanwendungen (DiGAs), haben, die von den gesetzlichen Krankenkassen erstattet werden. Besonders im Bereich der Rheumatologie bieten sich für das Management chronischer Erkrankungen und ihrer Komorbiditäten verschiedene Anknüpfungspunkte. Nicht nur unter rheumatologischen Patienten ist das Interesse an App-Angeboten groß, sondern auch unter deutschen Rheumatologen zeigt sich eine steigende Bereitschaft, Apps im Berufsalltag anzuwenden und Patienten zu empfehlen. Dieser Artikel will einen Überblick über die Entwicklung der App-Landschaft in der deutschsprachigen Rheumatologie vermitteln.

Schlüsselwörter

eHealth $\cdot$ mHealth · Digitalisierung .

Rheumatologie · Mobile Applikationen

\section{Mobile apps and their usage in rheumatology}

\section{Abstract}

The increasing use of smartphones is accompanied by a significant increase in the use of mobile applications (apps). Chronically ill patients could permanently profit from this development.This development is fuelled by the Digital Healthcare Act (DVG), whereby patients have a legal claim to certain apps, so-called digital health applications (DiGAs), which are reimbursed by the statutory health insurance companies. Especially in the field of rheumatology, there are various opportunities to implement apps in the management of chronic diseases and their comorbidities. Furthermore, rheumatic patients and rheumatologists are becoming interested in apps and are willing to use them in the daily routine. This article tries to shed light on the chances and risks of apps and gives a first insight into the digital landscape of rheumatology apps in Germany.

\section{Keywords}

eHealth - mHealth - Digital health . Rheumatology $\cdot$ Mobile applications

\section{Apps zum Krankheitsmonitoring}

Der quartalsweise Patientenbesuch erlaubt dem Rheumatologen nur eine begrenzte und punktuelle Einschätzung der Krankheitsaktivität. Mithilfe der Digitalisierung besteht die Möglichkeit einer kontinuierlichen Datenerhebung, die sowohl passiv als auch aktiv erfolgen kann. „Patient-reported outcomes“ (PROs) können bereits über verschiedenste Apps erfasst und übermittelt werden [21].

Vonseiten der Patienten scheint ebenfalls die Bereitschaft $\mathrm{zu}$ bestehen, sich an einer regelmäßigen Dateneingabe von 5 min wöchentlich zu beteiligen [14].

Neben der aktiven Datenerfassung durch den Patienten besteht grundsätz- lich auch die Möglichkeit, Daten passiv mittels Smartphone oder gekoppeltem Wearable (z. B. Smartwatch) zu erfassen. So konnten durch digitale Schrittzahlmessung in einer französischen Studie mit hoher Spezifität (97\%) und Sensitivität (96\%) Schübe bei Patienten mit RA und Spondyloarthritis (SpA) detektiert werden [22]. Weitere Anwendungsmöglichkeiten, wie z.B. die Messung der Fingerschwellung mittels Smartphonekamera [23], die objektive digitale Bestimmung der Wirbelsäulenbeweglichkeit von SpA-Patienten [24] und die Ermittlung der körperlichen Aktivität von Myositispatienten mittels Akzelerometermessung [25], werden ihren Einzug in den klinischen Alltag 


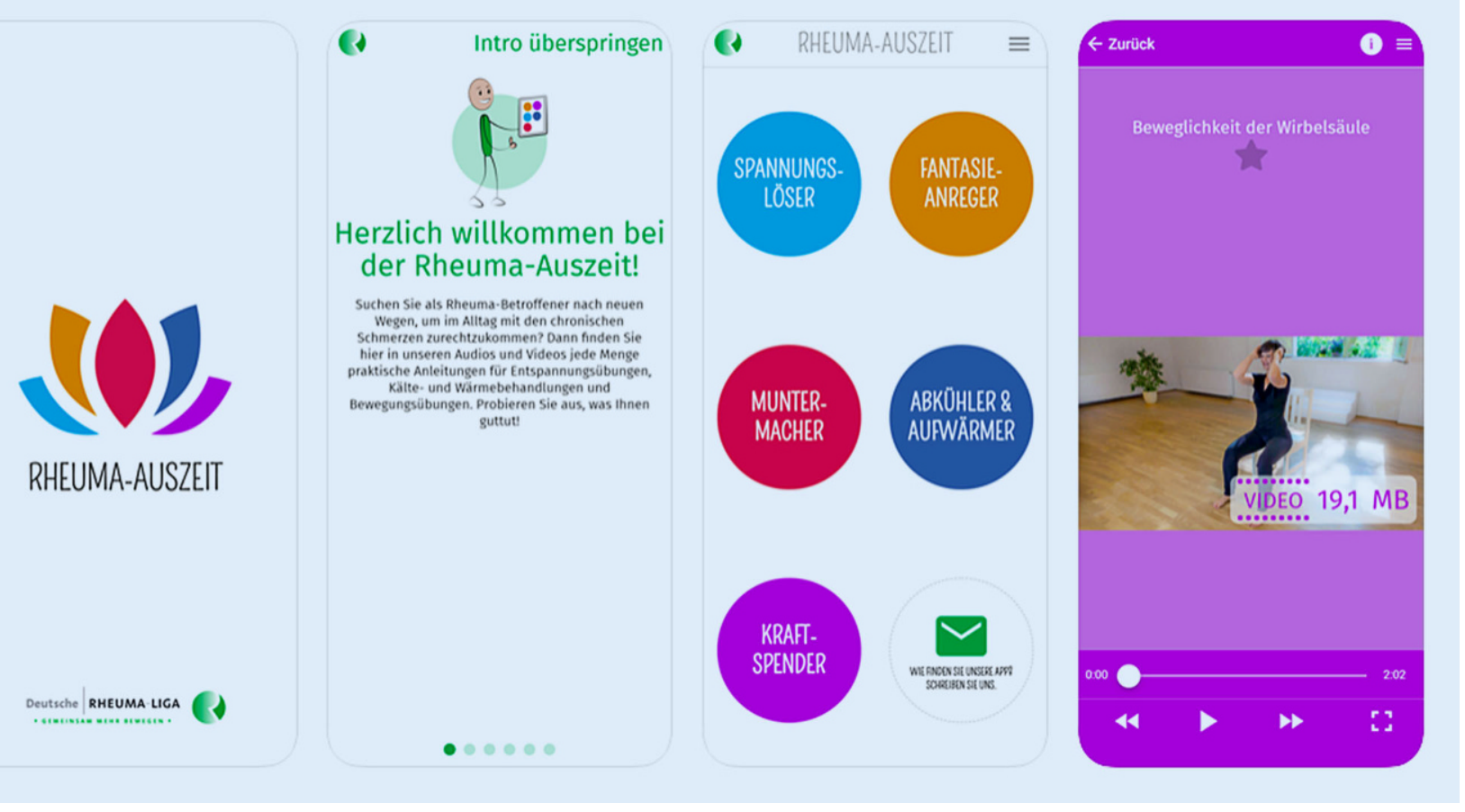

Abb. 2 \ Screenshots der App Rheuma-Auszeit (Deutsche Rheuma-Liga Bundesverband e.V.) [41]. (Mit freundl. Genehmigung $\odot$ Deutsche Rheuma-Liga Bundesverband e. V., alle Rechte vorbehalten)

finden und neue digitale Krankheitsmarker etablieren.

\section{Apps zur Symptomlinderung und Management von Komorbiditäten}

Bei vielen Patienten mit entzündlich rheumatischen Erkrankungen sind chronische Schmerzen und Depressionen wesentliche Komorbiditäten. Für die Behandlung von Depressionen mithilfe von Apps gibt es erste Studien mit Nutzennachweis [26].

Beispielsweise konnte in einer randomisierten kontrollierten Studie gezeigt werden, dass der Einsatz der Webapp HelloBetter mit einer signifikanten Reduktion von depressiven Symptomen assoziiert war [27, 28]. Die App Kaia gegen Rückenschmerzen sorgte in einer randomisiert kontrollierten Studie (Dauer 12 Wochen) für eine signifikant höhere Schmerzreduktion bei Patienten mit unspezifischen unteren Rückenschmerzen im Vergleich zu 6 Physiotherapieeinheiten und Online-Unterrichtsmaterial [29].

Physiotherapie, Ergotherapie und Sport sind für viele Rheumapatienten neben der Pharmakotherapie ebenfalls ein wichtiger Bestandteil der Therapie.
Für die Behandlung von chronischen unteren Rückenschmerzen gibt es bereits mehrere Apps, für die sich in Studien ein positiver Effekt nachweisen ließ $[29,30]$. In einer App-Analyse der Arbeitsgemeinschaft Junge Rheumatologie (AGJR) wurde die App Rheuma-Auszeit (Deutsche Rheuma-Liga Bundesverband e. V., - Abb. 2) am besten bewertet [21]. Die App richtet sich an Patienten mit entzündlich rheumatischen Erkrankungen und stellt Bewegungs- und Entspannungsübungen mittels Audio- und Videodateien zur Verfügung, die die Patienten sofort anwenden können.

Medizinische Apps können ebenfalls das Management von internistischen Komorbiditäten bei Patienten mit entzündlich rheumatischen Systemerkrankungen unterstützen. Insbesondere für den Diabetes mellitus finden sich inzwischen mehrere Apps, welche die Dokumentation von Blutzuckerwerten und Medikation im Tagebuchformat erleichtern und versuchen die Therapieadhärenz durch eine Erinnerungsfunktion zu verbessern. In Bezug auf harte Endpunkte (z.B. $\mathrm{HbA}_{1 c}$ ) erbrachten die wenigen bisher durchgeführten randomisierten Studien heterogene Ergebnisse
[31]. Ebenfalls untersucht ist der Einsatz von Apps bei Hypertoniepatienten. Eine systematische Übersichtsarbeit von $\mathrm{Li}$ et al. ergab günstige Effekte von Interventionen, die das Selbstmanagement von Patienten durch Apps unterstützten und mit einer Verringerung der Blutdruckwerte einhergingen [32].

\section{Apps als Informationsquelle und Nachschlagewerk}

Insbesondere für medizinisches Fachpersonal und Ärzte gibt es verschiedene Apps, die als mobile Informationsquelle und Nachschlagewerk verwendet werden können (z. B. Arzneimittelpocket 2018, Embryotox, Prime: PubMed Journals \& Tools, aerzteblatt.de, AMBOSS Wissen für Mediziner).

Viele Fachgesellschaften stellen mittlerweile ihre Leitlinien ebenfalls im App-Format zur Verfügung. So zeigt z. B. die Rheuma Schweiz Education App (• Abb. 3) Kurzvideos mit Anleitungen für Gelenkpunktionen. Die Österreichische Gesellschaft für Rheumatologie und Rehabilitation (ÖGR) hat mit dem RheumaGuide eine eigene App, die für die RA, PsA und SpA alle relevanten EULAR- 


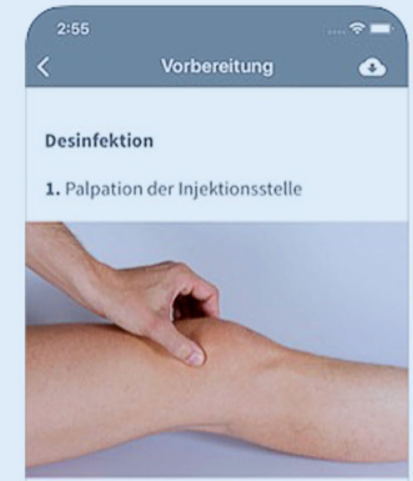

2. Markierung der Injektionsstelle mittels Abdruck in der Haut z.B. durch einen Kugelschreiber (Ring der Kugelschreiberspitze / Benutzen der Miene)

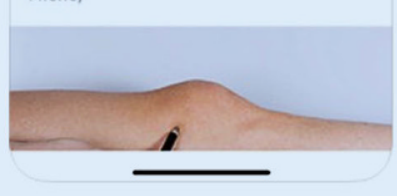

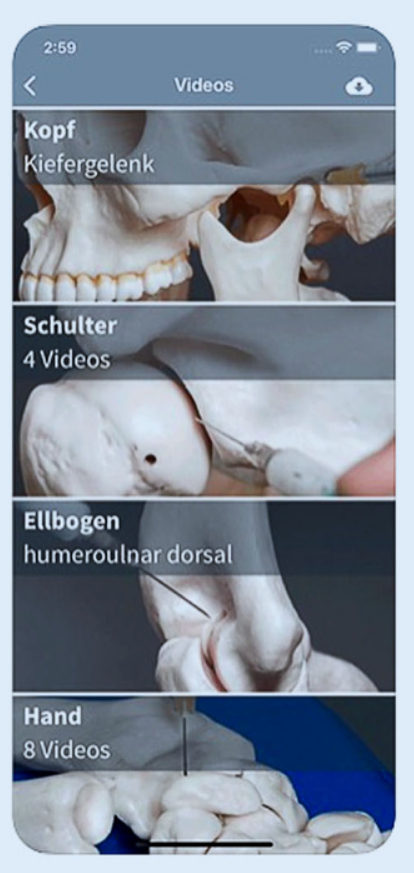

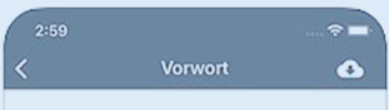

Geschätzte Kollegin, geschätzter Kollege

Bei guter Indikationsstellung bieten Injektionen oft den Schlüssel zur Behebung von lokalen Schmerzursachen, sei es eine Arthritis, eine Bursitis, eine Irritation von Båndern oder Sehnen oder die Kompression eines peripheren Nerven. Eine gute Injektionstechnik ist aber auch erforderlich für eine optimale Punktion eines Gelenkes zu diagnostischen Zwecken.

Die vorliegende App dient der Orientierung über vorbereitende Massnahmen bei Injektionen, aber auch über die anatomisch korrekt gesteuerte spezifische Injektion in Gelenke und Weichteile. Für die Indikation von Injektionen werden Kenntnisse der rheumatologischen Untersuchung und krankheitsrelevante Zusammenhänge vorausgesetzt. Diesbezüglich verweisen

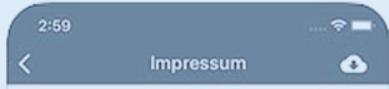

rheuma

(0 2017 Rheuma Schweiz

Autoren

Dr. med. Pius Brühlmann

Prof. Dr. med. Beat A. Michel

Dr. med. Martin Toniolo

Herausgeber

Rheuma Schweiz

www.rheuma-schweiz.ch

Konzeption und Realisation

Pomcanys Marketing AG

wwwipomcanys,ch

Rheuma Schweiz bedankt sich für die Unterstūtzung bei der Realisierung dieses Projektes bei Dr. med. Stella Schaffstein, Ashley Stutz und der Firma MSD Merck Sharp \& Dohme AG.

Abb. $3 \Delta$ Screenshots der App Rheuma Schweiz Education (Pomcany's Marketing AG) [42]. (Mit freundl. Genehmigung $\odot$ Rheuma Schweiz, alle Rechte vorbehalten)

Guidelines, Scorerechner, Schwangerschafts- und Impfinformationen sowie Links zu Patienteninformationsmaterial bereitstellt.

\section{Bewertung und Zertifizierung von rheumatologischen Apps}

Die App-Stores bieten ein umfangreiches Angebot mit täglich neu erscheinenden Apps. Allerdings sind viele Apps nur kurzzeitig in den Stores verfügbar oder aufgrund von technischen Updates nicht mehr adäquat nutzbar [33]. Aufgrund der geringen Vorschriften von App-Stores und kurzen Verweildauer vieler Apps ist es für Patienten und medizinisches Personal schwer, einen Eindruck von der Qualität der Apps zu gewinnen.

Aktuell existiert eine Vielzahl an AppBewertungsmöglichkeiten, wobei einige reinen Checklisten entsprechen und andere auf Punkteskalen basieren [34].

Zertifizierungen, Checklisten für Verbraucher oder Gütesiegel können ebenfalls Auskunft über die Qualität und Eignung einzelner Apps geben.

Eine 2018 veröffentlichte Analyse von Albrecht et al. zeigte, dass nur ein kleiner Anteil medizinischer Apps im deut- schen App-Store von Apple eine Zertifizierung aufweist [35]. Von 8767 identifizierten deutschsprachigen GesundheitsApps waren nur 41 mit einem Gütesiegel ausgezeichnet und 34 CE-zertifiziert. Fünf weitere nannten eines von 13 recherchierten nichtstaatlichen Gütesiegeln. Dazu zählten der Appcheck des Zentrums für Telematik und Telemedizin (ZTG), das HealthOn-Siegel, das DiaDigital-Siegel, EuroPriSe, HONcode, Test durch Stiftung Warentest, Trusted Apps, TÜV-Süd-Software-Prüfzeichen, Qualitätsprodukt Internetmedizin oder ePrivacy seal [36].

Eine Analyse der deutschsprachigen rheumatologischen Apps vom Juli 2018, die parallel im Google Play Store und Apple App Store verfügbar waren, ergab immerhin, dass 3 von 16 analysierten Apps CE-zertifiziert waren. Interessanterweise waren alle Apps vom selben Hersteller entwickelt worden (STAR Healthcare Management $\mathrm{GmbH}$ ) [21].

\section{Evidenz}

Obwohl die Anzahl von Publikationen zu Apps in der Rheumatologie in den letzten Jahren stetig gestiegen ist, besteht für den Nutzen von rheumatologischen Apps aktuell noch sehr begrenzte Evidenz. Studien, die die Wirksamkeit von deutschsprachigen rheumatologischen Apps belegen, liegen aktuell nicht vor [21]. Najm et al. konnten in einem systematischen Review von 2019 ebenfalls nur 2 randomisiert kontrollierte klinische Studien für Apps in der Rheumatologie identifizieren [33]: In der einen Studie konnten bei Osteoarthrosepatienten mittels AppNutzung eine signifikante Erhöhung der Schrittzahl pro Tag sowie eine Reduktion der Schmerzen gezeigt werden [37]. Die Studie von Kristjansdottir et al. umfasste eine per Smartphone durchgeführte Intervention mit Tagebuch und personalisiertem Feedback an Patienten mit Fibromyalgie. Hier konnte für die Interventionsgruppe im Follow-up nach 11 Monaten eine signifikante Reduktion im Catastrophizing Score nachgewiesen werden [38].

Eine Pilotstudie von Mary et al. konnte eine Verbesserung der Therapieadhärenz (Einnahme von Methotrexat) durch SMS-Erinnerungen bei RA-Patienten belegen [39]. 
Tab. 1 EULAR Points to consider für die Entwicklung von rheumatologischen Apps nach Najm et al. [40]

1. Der Informationsgehalt in Selbstmanagement-Apps sollte aktuell, wissenschaftlich begründbar, für den Benutzer vertretbar und, wenn möglich, evidenzbasiert sein

2. Apps sollten relevant und auf die individuellen Bedürfnisse von Menschen mit rheumatischen und muskuloskeletalen Erkrankungen zugeschnitten sein

3. An der Gestaltung, Entwicklung und Validierung von Selbstmanagement-Apps sollten Patienten mit rheumatischen und muskuloskeletalen Erkrankungen sowie die relevanten medizinischen Fachgruppen beteiligt werden

4. Es sollte Transparenz bezüglich des Entwicklers einer App, der Finanzierungsquelle, des Inhaltsvalidierungsprozesses, des Versions-Updates und des Datenschutzes bestehen

5. Die Datenerfassung als Teil von Apps muss alle anwendbaren gesetzlichen Rahmenbedingungen, insbesondere den Datenschutz, erfüllen

6. Apps dürfen bei Patienten mit rheumatischen und muskuloskeletalen Erkrankungen nicht zu physischen oder emotionalen Schäden führen

7. Apps können die Kommunikation zwischen Patienten und Gesundheitsdienstleistern erleichtern und zu elektronischen Gesundheitsakten oder zur Forschung beitragen

8. Beim Design von Apps sollte die Zugänglichkeit für Patienten mit rheumatischen und muskuloskeletalen Erkrankungen über alle Altersgruppen und Fähigkeiten hinweg berücksichtigt werden

9. Wenn ein soziales Netzwerk ein wichtiger Bestandteil einer App ist, sollten Strukturen vorhanden sein, die eine angemessene inhaltliche Moderation gewährleisten

10. Die rheumatologische Gemeinschaft sollte das Kosten-Nutzen-Verhältnis von Apps vor der Befürwortung und/oder Bewerbung prüfen

Tab. 2 Übersicht der diskutierten Apps

\begin{tabular}{|c|c|c|c|c|c|c|c|}
\hline App-Name & Entwickler & Zweck & Zielgruppe & Erkrankung & Preis & iOS & Android \\
\hline ABATON & ABATON GmbH & Verlaufsmonitoring & $\begin{array}{l}\text { Ärzte \& Pati- } \\
\text { enten }\end{array}$ & $\begin{array}{l}\text { Rheum. Formen- } \\
\text { kreis }\end{array}$ & Kostenlos & Ja & $\mathrm{Ja}$ \\
\hline Ada & Ada Health & $\begin{array}{l}\text { Diagnose Unterstüt- } \\
\text { zung }\end{array}$ & Patienten & - & Kostenlos & Ja & Ja \\
\hline RhePort & $\begin{array}{l}\text { Verein zur Förderung der Rheuma- } \\
\text { tologie e.V. }\end{array}$ & $\begin{array}{l}\text { Patientenedukation } \\
\text { Terminvergabe }\end{array}$ & $\begin{array}{l}\text { Patienten \& } \\
\text { Ärzte }\end{array}$ & $\begin{array}{l}\text { Rheum. Formen- } \\
\text { kreis }\end{array}$ & Kostenlos & b & b \\
\hline Rheuma-VOR & Universitätsmedizin Mainz & $\begin{array}{l}\text { Diagnose Unterstüt- } \\
\text { zung }\end{array}$ & Ärzte & RA, PsA, AxSpa & Kostenlos & Ja & $\mathrm{Ja}$ \\
\hline HelloBetter & $\begin{array}{l}\text { GET.ON Institut für Online-Ge- } \\
\text { sundheitstrainings GmbH } 2020\end{array}$ & Psychotherapie & Patienten & $\begin{array}{l}\text { Psychische } \\
\text { Erkrankungen }\end{array}$ & a & b & b \\
\hline $\begin{array}{l}\text { Kaia gegen Rücken- } \\
\text { schmerzen }\end{array}$ & Kaia Health Software GmbH & Therapie & Patienten & $\begin{array}{l}\text { Rücken- } \\
\text { schmerzen }\end{array}$ & Kostenlos & Ja & Ja \\
\hline Rheuma-Auszeit & $\begin{array}{l}\text { Deutsche Rheuma-Liga Bundes- } \\
\text { verband e.V. }\end{array}$ & Schmerzbewältigung & Patienten & $\begin{array}{l}\text { Rheum. Formen- } \\
\text { kreis }\end{array}$ & Kostenlos & Ja & Ja \\
\hline $\begin{array}{l}\text { Arzneimittelpocket } \\
2018\end{array}$ & Börm Bruckmeier Verlag GmbH & Nachschlagewerk & Ärzte & - & Kostenlos & Ja & Ja \\
\hline Embryotox & Charité Universitätsmedizin Berlin & Nachschlagewerk & Ärzte & - & Kostenlos & Ja & Ja \\
\hline $\begin{array}{l}\text { Prime: PubMed } \\
\text { Journals \& Tools }\end{array}$ & Unbound Medicine, Inc & Nachschlagewerk & Ärzte & - & Kostenlos & Ja & $\mathrm{Ja}$ \\
\hline Aerzteblatt.de & Deutscher-Ärzte-Verlag GmbH & Nachschlagewerk & Ärzte & - & Kostenlos & Ja & Ja \\
\hline $\begin{array}{l}\text { AMBOSS Wissen für } \\
\text { Mediziner }\end{array}$ & AMBOSS GmbH & Nachschlagewerk & Ärzte & - & Kostenlos $^{\mathrm{a}}$ & Ja & $\mathrm{Ja}$ \\
\hline $\begin{array}{l}\text { Rheuma Schweiz } \\
\text { Education }\end{array}$ & Pomcany's Marketing AG & Nachschlagewerk & Ärzte & $\begin{array}{l}\text { Rheum. Formen- } \\
\text { kreis }\end{array}$ & Kostenlos ${ }^{\mathrm{a}}$ & $\mathrm{Ja}$ & Ja \\
\hline RheumaGuide & $\begin{array}{l}\text { MedMedia Verlag und Mediaser- } \\
\text { vice GmbH }\end{array}$ & Nachschlagewerk & Ärzte & $\begin{array}{l}\text { Rheum. Formen- } \\
\text { kreis }\end{array}$ & Kostenlos & Ja & Ja \\
\hline \multicolumn{8}{|c|}{$\begin{array}{l}R A \text { rheumatoide Arthritis, PsA Psoriasisarthritis, AxSpa axiale Spondyloarthritis } \\
\text { aLizenz bzw. In-App-Käufe zur Nutzung notwendig } \\
\text { 'Webapp }\end{array}$} \\
\hline
\end{tabular}




\section{Empfehlungen für die Entwicklung und Nutzung von Apps}

Für die Entwicklung und Nutzung neuer Apps im Bereich der Rheumatologie hat die EULAR 2019 Empfehlungen ausgesprochen (『 Tab. 1; [40]).

Neben diesen Empfehlungen zeigte die Studie der AGJR, dass Apps mit Multimedia-Inhalten besser bewertet wurden und es für seltene rheumatologische Erkrankungen wenige App-Angebote gab [21].

Die - Tab. 2 zeigt eine Übersicht der diskutierten Apps.

\section{Fazit für die Praxis}

\section{- Die Mehrheit der Patienten ist bereit, Daten für Forschungszwecke zur Verfügung zu stellen, und offen für die Nutzung medizinischer Apps. \\ - Patienten haben einen Anspruch auf DiGAs, und Rheumatologen können indikationsspezifisch verordnen. \\ - Aktuell gibt es nur wenige Studien, die den Nutzen entsprechender Apps untersuchen. \\ - Die DGRh-Kommission „Digitale Rheumatologie" wurde gegründet, um den digitalen Wandel aktiv zu gestalten.}

\section{Korrespondenzadresse}

\section{Dr. M. Krusche}

Medizinische Klinik mit Schwerpunkt Rheumatologie und Klinische Immunologie, Charité Universitätsmedizin Charitéplatz 1, 10117 Berlin, Deutschland martin.krusche@charite.de

\section{Dr. J. Knitza, MHBA}

Medizinische Klinik 3 - Rheumatologie und Immunologie, Universitätsklinikum Erlangen, Friedrich-Alexander-Universität ErlangenNürnberg (FAU)

Ulmenweg 18, 91054 Erlangen, Deutschland johannes.knitza@uk-erlangen.de

Funding. Open Access funding provided by Projekt DEAL.
Einhaltung ethischer Richtlinien

Interessenkonflikt. M. Krusche, N. Ruffer und J. Knitza geben an, dass kein Interessenkonflikt besteht. M. Grahammer ist Gründer der ABATON GmbH.

Für diesen Beitrag wurden von den Autoren keine Studien an Menschen oder Tieren durchgeführt. Für die aufgeführten Studien gelten die jeweils dort angegebenen ethischen Richtlinien.

Open Access. Dieser Artikel wird unter der Creative Commons Namensnennung 4.0 International Lizenz veröffentlicht, welche die Nutzung, Vervielfältigung, Bearbeitung, Verbreitung und Wiedergabe in jeglichem Medium und Format erlaubt, sofern Sie den/die ursprünglichen Autor(en) und die Quelle ordnungsgemäßnennen, einen Link zur Creative Commons Lizenz beifügen und angeben, ob Änderungen vorgenommen wurden.

Die in diesem Artikel enthaltenen Bilder und sonstiges Drittmaterial unterliegen ebenfalls der genannten Creative Commons Lizenz, sofern sich aus der Abbildungslegende nichts anderes ergibt. Sofern das betreffende Material nicht unter der genannten Creative Commons Lizenz steht und die betreffende Handlung nicht nach gesetzlichen Vorschriften erlaubt ist, ist für die oben aufgeführten Weiterverwendungen des Materials die Einwilligung des jeweiligen Rechteinhabers einzuholen.

Weitere Details zur Lizenz entnehmen Sie bitte der Lizenzinformation auf http://creativecommons.org/ licenses/by/4.0/deed.de.

\section{Literatur}

1. Brandt M (2019) Smartphones In Deutschland. https://de.statista.com/infografik/17190/ ausgewaehlte-daten-zur-smartphone-nutzung in-deutschland/.Zugegriffen: 05.04.2020

2. HealthOn (2020) HealthOn Statistiken. https:// www.healthon.de/healthon-statistiken. Zugegriffen: 5.Apr. 2020

3. statista (2020) An welche Zielgruppen richtet sich Ihre mHealth-App hauptsächlich? https://de.statista.com/statistik/daten/studie/ 695482/umfrage/umfrage-unter-mhealth-appentwicklern-zur-den-wichtigsten-zielgruppen/ statista. Zugegriffen:03.04.2020

4. Bundesgesetzblatt (2019) Gesetz für eine bessere Versorgung durch Digitalisierung und Innovation (Digitale-Versorgung-Gesetz - DVG). https://www.bgbl.de/xaver/ bgbl/start.xav?startbk=Bundesanzeiger_BGBI\& jumpTo=bgbl119s2562.pdf\#_bgbl_\%2F\%2F* \%5B\%40attr_id\%3D\%27bgbl119s2562.pdf\%27 \%5D_1585489262195.Zugegriffen:03.04.2020

5. DGIM (2017) DGIM im Gespräch:Professor Dr.med Gerd Hasenfuß zur neuen Task Force mHealth. https://www.dgim.de/fileadmin/user_upload/ PDF/UEber_uns/Gremien/2017_Interview_ mobilehealth.pdf.Zugegriffen:05.04.2020

6. Hirsch MC, Ronicke S, Krusche M, Wagner AD (2020) Rare diseases 2030: how augmented Al will support diagnosis and treatment of rare diseases in the future. Ann Rheum Dis. https://doi.org/10. 1136/annrheumdis-2020-217125

7. Ronicke S, Hirsch MC, Turk E, Larionov K, Tientcheu D, Wagner AD (2019) Can a decision support system accelerate rare disease diagnosis? Evaluating the potential impact of Ada DX in a retrospective study. Orphanet J Rare Dis 14(1):69. https://doi.org/10.1186/s13023-019-1040-6

8. Krusche M, Klemm P, Mucke J, Vossen D, Kleyer A, Sewerin P, Knitza J (2020) Electronic patient-reported outcomes: a survey about acceptance, usage and barriers among German rheumatologists. JMIR Prepr. https://doi.org/10. 2196/preprints.18117

9. Wickström HL, Oien RF, Fagerstrom C, Anderberg P, Jakobsson U, Midlov PJ (2018) Comparing video consultation with inperson assessment for Swedish patients with hard-to-heal ulcers: registry-based studies of healing time and of waiting time. BMJ Open 8(2):e17623. https://doi. org/10.1136/bmjopen-2017-017623

10. Kramer U (2017) Wie gut sind Gesundheits-Apps? Aktuel Ernahrungsmed 42(03):193-205. https:// doi.org/10.1055/s-0043-109130

11. Medizinprodukte BfAu (2020) Das Fast-TrackVerfahren für digitale Gesundheitsanwendungen (DiGA) nach § 139e SGB V - Ein Leitfaden für Hersteller, Leistungserbringer und Anwender. https://www.bfarm.de/SharedDocs/Downloads/ DE/Service/Beratungsverfahren/DiGA-Leitfaden. pdf?_blob=publicationFile\&v=1. Zugegriffen: 28. Apr. 2020

12. Knitza J, Vossen D, Geffken I, Krusche M, Meyer M, Sewerin P, Kleyer A, Hueber AJ, Arbeitskreis Junge Rheumatologen (2019) Nutzung von MedizinApps und Online-Plattformen unter deutschen Rheumatologen: Ergebnisse der rheumadocsRecherche und DGRh-Kongress-Umfragen von 2016 und 2018. Z Rheumatol 78(9):839-846. https://doi.org/10.1007/s00393-018-0578-3

13. Nikiphorou E, Studenic P, Ammitzboll CG, Canavan $M$, Jani M, Ospelt C, Berenbaum F (2017) Social media use among young rheumatologists and basic scientists: Results of an international survey by the Emerging EULAR Network (EMEUNET). Ann Rheum Dis 76(4):712-715. https://doi.org/10. 1136/annrheumdis-2016-209718

14. Knitza J, Raab C, Lambrecht A, Simon D, Hagen $M$, Bayat S, Schett G, Kleyer A, Hueber A (2019) The urge for mobile apps in rheumatology-A German patient perspective. 2019 ACR/ARP Annual Meeting.

15. Costello R, Jacklin C, Jameson Evans M, McBeth J, Dixon WG (2018) Representativeness of a digitally engaged population and a patient organisation population with rheumatoid arthritis and their willingness to participate in research: a crosssectional study. RMD Open 4(1):e664. https://doi. org/10.1136/rmdopen-2018-000664

16. Raza K, Stack R, Kumar K, Filer A, Detert J, Bastian H, Burmester GR, Sidiropoulos P, Kteniadaki E, Repa A, Saxne T, Turesson C, Mann H, Vencovsky J, Catrina A, Chatzidionysiou A, Hensvold A, RantapaaDahlqvist S, Binder A, Machold K, Kwiatkowska B, Ciurea A, Tamborrini G, KyburzD, Buckley CD (2011) Delays in assessment of patients with rheumatoid arthritis: variations across Europe. Ann Rheum Dis 70(10):1822-1825. https://doi.org/10.1136/ard. 2011.151902

17. Feuchtenberger $M$, Nigg AP, Kraus MR, Schafer A (2016) Rate of proven rheumatic diseases in a large collective of referrals to an outpatient rheumatology clinic under routine conditions. Clin Med Insights Arthritis Musculoskelet Disord 9:181-187. https://doi.org/10.4137/CMAMD. S40361

18. Urruticoechea-Arana A, Leon-Vazquez $F$, GinerRuiz V, Andreu-Sanchez JL, Olive-Marques A, 
Freire-Gonzalez M, Pego-Reigosa JM, MunozFernandez S, Roman-Ivorra JA, Alegre-Sancho JJ, Vargas-Negrin F, Medina-Abellan M, Cobo-lbanez T, Mas-Garriga X, Calvo-Alen J, Costa-Ribas $C$, Blanco-Vela R, Perez-Martin A, Beltran-Catalan E, Forcada-Gisbert J, Hernandez-Miguel MV, Hermosa-Hernan JC, Narvaez-Garcia J, Nieto-Pol E, Rua-Figueroa I (2019) Development of an application for mobile phones (App) based on the collaboration between the Spanish Society of Rheumatology and Spanish Society of Family Medicine for the referral of systemic autoimmune diseases from primary care to rheumatology. Reumatol Clin. https://doi.org/10.1016/j.reuma. 2019.09.001

19. Knitza J, Knevel R, Raza K, Bruce T, EimerE, Gehring I Mathsson-Alm L, Poorafshar M, Hueber A, Schett G, Johannesson M, Catrina M, Klareskog L (2020) Towards earlier diagnosis in rheumatology using combined eHealth tools: The JPAST Project. JMIR Mhealth Uhealth. https://doi.org/10.2196/17507

20. Powley L, Mcllroy G, Simons G, Raza K (2016) Are online symptoms checkers useful for patients with inflammatory arthritis? BMC Musculoskelet Disord 17(1):362. https://doi.org/10.1186/s12891-0161189-2

21. Knitza J, Tascilar K, Messner EM, Meyer M, Vossen D, Pulla A, Bosch P, Kittler J, Kleyer A, Sewerin P, Mucke J, Haase I, Simon D, Krusche M (2019) German mobile apps in rheumatology: Review and analysis using the mobile application rating scale (MARS). JMIR Mhealth Uhealth 7(8):e14991. https://doi.org/10.2196/14991

22. Gossec L, Guyard F, Leroy D, Lafargue T, Seiler M, Jacquemin C, Molto A, Sellam J, Foltz V, Gandjbakhch F, Hudry C, Mitrovic S, Fautrel B, Servy H (2019) Detection of flares by decrease in physical activity, collected using wearable activity trackers in rheumatoid arthritis or axial spondyloarthritis: An application of machine learning analyses in rheumatology. Arthritis Care Res (Hoboken) 71(10):1336-1343. https://doi.org/10.1002/acr. 23768

23. Mollard E, Michaud K (2018) A mobile app with optical imaging for the self-management of hand rheumatoid arthritis: pilot study. JMIR Mhealth Uhealth 6(10):e12221. https://doi.org/10.2196/ 12221

24. Kiefer D, Baraliakos X, Buhring B, Kiltz U, Braun J (2020) Epionics SPINE - Anwendung einer objektiven Untersuchungsmethode der Wirbelsäulenbeweglichkeit bei Patienten mit axialer Spondyloarthritis. Z Rheumatol 79(2):143-152. https://doi.org/10.1007/s00393-019-00692-3

25. Oldroyd A, Little MA, Dixon W, Chinoy H (2019) A review of accelerometer-derived physical activity in the idiopathic inflammatory myopathies. BMC Rheumatol 3:41. https://doi.org/10.1186/s41927019-0088-1

26. Mohr DC, Tomasino KN, Lattie EG, Palac HL, Kwasny MJ, Weingardt K, Karr CJ, Kaiser SM, Rossom RC, Bardsley LR, Caccamo L, StilesShields C, Schueller SM (2017) Intellicare: an eclectic, skills-based app suite for the treatment of depression and anxiety. J Med Internet Res 19(1):e10. https://doi.org/10.2196/jmir.6645

27. Nobis S, Lehr D, Ebert DD, Baumeister H, Snoek F, Riper H, Berking M (2015) Efficacy of a webbased intervention with mobile phone support in treating depressive symptoms in adults with type 1 and type 2 diabetes: a randomized controlled trial. Diabetes Care 38(5):776-783. https://doi.org/10. 2337/dc14-1728
28. Ebert DD, Nobis $\mathrm{S}$, Lehr $\mathrm{D}$, Baumeister $\mathrm{H}$, Riper $\mathrm{H}_{\text {, }}$ Auerbach RP, Snoek F, Cuijpers P, Berking M (2017) The 6-month effectiveness of Internetbased guided self-help for depression in adults with Type 1 and 2 diabetes mellitus. Diabet Med 34(1):99-107. https://doi.org/10.1111/dme. 13173

29. Toelle TR, Utpadel-Fischler DA, Haas KK, Priebe JA (2019) App-based multidisciplinary back pain treatment versus combined physiotherapy plus online education: a randomized controlled trial. NPJ Digit Med 2:34. https://doi.org/10.1038/ s41746-019-0109-x

30. Chhabra HS, Sharma S, Verma S (2018) Smartphone app in self-management of chronic low back pain: a randomized controlled trial. Eur Spine J 27(11):2862-2874. https://doi.org/10. 1007/s00586-018-5788-5

31. Fleming GA, Petrie JR, Bergenstal RM, Holl RW, Peters AL, Heinemann L (2020) Diabetes digital app technology: benefits, challenges, and recommendations. A consensus report by the European Association for the Study of Diabetes (EASD) and the American Diabetes Association (ADA) diabetes technology working group. Diabetes Care 43(1):250-260. https://doi.org/10.2337/dci190062

32. Li R, Liang N, Bu F, Hesketh T (2020) The effectiveness of self-management of hypertension in adults using mobile health: systematic review and meta-analysis. JMIR Mhealth Uhealth 8(3):e17776. https://doi.org/10.2196/17776

33. Najm A, Gossec L, Weill C, Benoist D, Berenbaum F, Nikiphorou E (2019) Mobile health apps for selfmanagement of rheumatic and musculoskeletal diseases: systematic literature review. JMIR Mhealth Uhealth 7(11):e14730. https://doi.org/10. 2196/14730

34. Nouri R, Kalhori SRN, Ghazisaeedi M, Marchand G, Yasini M (2018) Criteria for assessing the quality of mHealth apps: a systematic review. J Am Med Inform Assoc 25(8):1089-1098. https://doi.org/10. 1093/jamia/ocy050

35. Albrecht UV, Hillebrand U, von Jan U (2018) Relevance of trust marks and CE labels in Germanlanguage store descriptions of health apps: analysis. JMIR Mhealth Uhealth 6(4):e10394. https://doi.org/10.2196/10394

36. aerzteblatt.de (2018) Die wenigstens MedizinApps werben mit Gütesiegeln oder einer CEKennzeichnung. https://www.aerzteblatt.de/ nachrichten/94909/Die-wenigstens-MedizinApps-werben-mit-Guetesiegeln-oder-einer-CEKennzeichnung.Zugegriffen:05.04.2020

37. Skrepnik N, Spitzer A, Altman R, Hoekstra J, Stewart J, Toselli R (2017) Assessing the impact of a novel smartphone application compared with standard follow-up on mobility of patients with knee osteoarthritis following treatment with Hylan G-F 20: a randomized controlled trial. JMIR Mhealth Uhealth 5(5):e64. https://doi.org/10. 2196/mhealth.7179

38. Kristjansdottir OB, Fors $E A$, Eide $E$, Finset $A$ StensrudTL, vanDulmen S, Wigers SH, EideH(2013) A smartphone-based intervention with diaries and therapist feedback to reduce catastrophizing and increase functioning in women with chronic widespread pain. part 2: 11-month follow-up results of a randomized trial. J Med Internet Res 15(3):e72.https://doi.org/10.2196/jmir.2442

39. Mary A, Boursier A, Desailly Henry I, Grados F, Sejourne A, Salomon S, Fardellone P, Brazier M, Goeb V (2019) Mobile phone text messages and effect on treatment adherence in patients taking methotrexate for rheumatoid arthritis: a randomized pilot study. Arthritis Care Res (Hoboken) 71(10):1344-1352. https://doi.org/10. 1002/acr.23750

40. Najm A, Nikiphorou E, Kostine M, Richez C, Pauling JD, Finckh A, RitschI V, Prior Y, Balazova $P$, Stones $S$, Szekanecz Z, lagnocco A, Ramiro $S$, Sivera F, Dougados M, Carmona L, Burmester G, Wiek D, Gossec L, Berenbaum F (2019) EULAR points to consider for the development, evaluation and implementation of mobile health applications aiding self-management in people living with rheumatic and musculoskeletal diseases. RMOpen 5(2):e1014. https://doi.org/10.1136/rmdopen2019-001014

41. Apple (2020) App Store-Vorschau RheumaAuszeit. https://apps.apple.com/de/app/rheumaauszeit/id1068203647.Zugegriffen: 5. Apr. 2020

42. Apple (2020) App Store-Vorschau Rheuma Schweiz Education. https://apps.apple.com/ch/ app/rheuma-schweiz-education/id911358609. Zugegriffen:5.Apr. 2020 